\title{
The effects of smoking and drinking on all-cause mortality in patients with dilated cardiomyopathy: a single-center cohort study
}

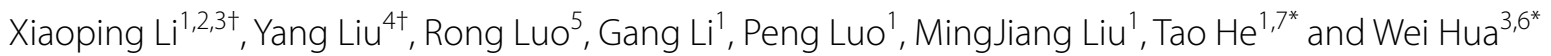

\begin{abstract}
Subject: Recent studies have shown that smoking and drinking are associated with poorer outcomes in patients with cardiomyopathy. The purpose of this study was to determine all-cause mortality in dilated cardiomyopathy (DCM) associated with smoking and drinking.

Methods: An observational cohort study was undertaken in DCM patients from November 2003 to September 2011. A total of 1118 patients were enrolled, with a mean follow-up of $3.5 \pm 2.3$ years. Standard demographics were obtained, and transthoracic echocardiography and routine blood testing were performed shortly after admission. Outcome assessment was based on the all-cause death after admission.

Results: The patients were divided into three groups: non-smokers $(n=593)$, mild-to-moderate smokers $(n=159)$ and heavy smokers $(n=366)$. The all-cause mortality rates showed no differences between the three groups (23.8, 20.8 and $24 \%$, respectively; log-rank $\left.\chi^{2}=1.281, P=0.527\right)$. There was also no significant difference in mortality between non-drinkers $(n=747)$, mild drinkers $(n=142)$ and moderate drinkers $(n=229)(23.7,23.2$ and $22.3 \%$, respectively; log-rank $\chi^{2}=2.343, P=0.310$ ). In the Cox analysis, neither the smoking (HR 0.971, $P=0.663$ ) nor the drinking status (HR 0.891, $P=0.140$ ) was a significant independent predictor of all-cause mortality in patients with DCM.

Conclusion: In conclusion, there were no significant differences in mortality between the smoking- and drinkingrelated patient groups, indicating no effect of smoking and drinking on all-cause mortality in patients with DCM in the present large-scale study.
\end{abstract}

Keywords: Smoking, Drinking, Dilated cardiomyopathy, All-cause mortality, Survival

\section{Background}

Dilated cardiomyopathy (DCM), a disease of the heart muscle characterized by ventricular dilatation and impaired systolic function, is the third most common cause of heart failure $[1,2]$. The prognosis of patients with DCM is poor, with approximately half of the patients dying within 5 years of diagnosis, and it is necessary for

\footnotetext{
*Correspondence: hch0119@sina.com; drhua@aliyun.com ${ }^{\dagger}$ Xiaoping Li and Yang Liu contributed equally to this work

${ }^{6}$ Cardiovascular Institute and Fuwai Hospital, Chinese Academy

of Medical Sciences, Peking Union Medical College, Beijing 100037, China

${ }^{7}$ Hospital of the University of Electronic Science and Technology of China

and Sichuan Provincial People's Hospital, Chengdu 610072, China

Full list of author information is available at the end of the article
}

the physician to predict which clinical course an individual patient may follow $[1,2]$.

Studies in mice and humans have shown that alcohol is a direct myocardial toxin that causes ultrastructural damage. Heavy drinking has been associated with left ventricular dysfunction and DCM, referred to as alcoholic cardiomyopathy [3, 4]. According to most studies, heavy drinking is associated with increased cardiovascular morbidity and mortality [5-7]. The US National Health and Nutrition Examination has stated that alcohol consumption has a linear relationship with mortality, with a slightly higher mortality risk for even light drinking $[6,7]$. However, some studies have shown that moderate alcohol consumption has cardioprotective effects; 
reduces the risk of chronic heart failure, coronary artery disease and stroke; and decreases cardiovascular and allcause mortality [8-12].

Cigarette smoking is a major modifiable risk factor for cardiovascular diseases, including coronary artery disease, stroke, peripheral vascular disease and congestive heart failure $[13,14]$. Both smoking and exposure to passive smoke are major preventable causes of cardiovascular morbidity and mortality $[15,16]$. Previous studies have indicated that smoking is related to cardiomyopathy [17] and is an important risk factor for idiopathic congestive cardiomyopathy [18]. However, recent studies have suggested that patients with DCM who smoke have a better prognosis than that of nonsmokers [19-21], although data from New Zealand show that smoking is associated with poorer survival in patients with DCM [22].

Although heavy drinking and smoking are known risk factors for cardiovascular mortality, their roles in DCM patients remain unclear $[5,19-22]$. Therefore, in the present study, we aimed to evaluate the association of drinking and smoking with all-cause mortality in hospitalized patients with DCM in China.

\section{Subjects and methods}

\section{Patients and follow-up}

A retrospective, observational cohort study of DCM patients was conducted from November 2003 to September 2011. The patients were admitted with symptoms of decompensation and physical signs of heart failure, and DCM was defined as systolic dysfunction (LVEF $\leq 50 \%$ ) with LV dilation in the absence of an apparent secondary cause of cardiomyopathy [23]. Of the 1317 enrolled patients, 175 patients were excluded from the study owing to the presence of various secondary cardiomyopathies, and data on smoking and drinking were lacking for 24 and 25 patients, respectively (Fig. 1). Thus, the final analysis included 1118 patients, with data on drinking lacking for 1 patient. The end point of the study was the all-cause mortality, which was assessed for all patients based on medical records and medical follow-up calls. Mortality data were obtained for all study patients from hospitalization to death. Data from patients who underwent cardiac transplantation were censored at the time of transplantation, if alive, to the date of the most recent clinical evaluation. The mean follow-up was $3.5 \pm 2.3$ years. The institutional review board approval was obtained.

\section{Smoking and drinking habits}

The three categories of drinkers were: non-drinkers; mild drinkers, who reported drinking an average of one drink a day on the days on which they consumed alcohol during the previous year; and moderate drinkers, who reported

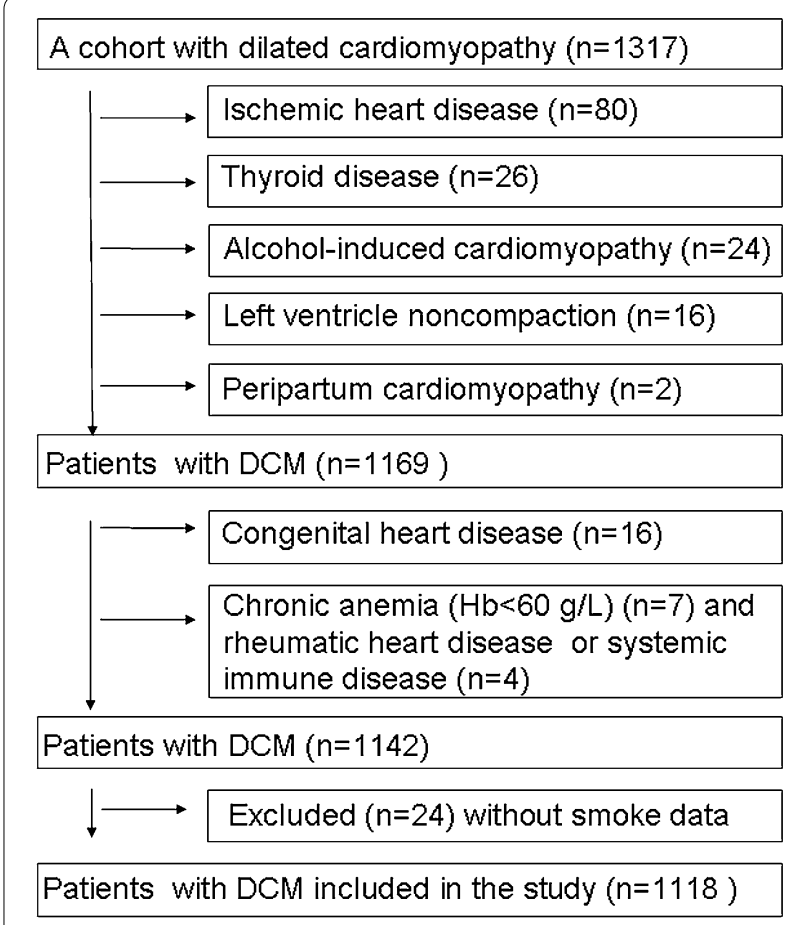

Fig. 1 Flowchart for participants involved in the present study

drinking an average of two drinks a day on the days on which they consumed alcohol during the previous year (1 drink $=12 \mathrm{~g}$ alcohol) [24]. Three categories of smokers were considered: non-smokers, mild-to-moderate smokers, who had smoked less than 40-pack years (PY); and heavy smokers, who had smoked $\geq 40$ PY [25]. PY are calculated as the number of cigarettes smoked per day multiplied by the years of smoking, divided by 20 [26].

\section{Echocardiography}

Patients were imaged in the left lateral decubitus position using a commercially available system equipped with a $3.5 \mathrm{MHz}$ transducer. Two-dimensional grayscale, pulsed, continuous and color Doppler data were acquired from the parasternal and apical views. For tissue Doppler imaging, the sector width was adjusted to obtain a frame rate of at least 115 frames/s. Left ventricular ejection fraction (LVEF) was calculated using Simpson's biplane technique [27].

\section{Statistical analysis}

The continuous variables are expressed as mean \pm SD or as medians and interquartile ranges. The categorical variables between groups were compared using Chi-square tests. Hazard ratios with $95 \%$ confidence intervals (95\% CIs) were used to estimate the adjusted relative risk for the various groups. Kaplan-Meier survival curves were 
compared using the log-rank test. Multivariate Cox proportional hazards regression models were applied to adjust for any confounding variables among groups. The analyses were conducted using SPSS (version 16.0, SPSS Inc., Chicago, IL, USA), and all tests were two sided. A $p$ value $<0.05$ was used to determine statistical significance.

\section{Results}

\section{Characteristics of the study population}

The cohort consisted of 1118 patients with DCM: 300 (26.8 \%) women and 818 (73.2 \%) men, with a mean age of $51.2 \pm 14.6$ years. Among the 1118 patients, those of Han nationality were 1074 (96.1\%), while minor nationality were 44 (3.9\%); patients who lived in the north of the Yazi River were 970 (86.8 \%) and those who lived in the south of the Yazi River were 148 (13.2\%). Of these subjects, $53.1 \%(n=593)$ were non-smokers, $14.2 \%$ $(n=159)$ were mild-to-moderate smokers and $32.7 \%$ $(n=366)$ were heavy smokers. In terms of drinking, $66.9 \%(n=747)$ of the cohort were non-drinkers; $12.7 \%$ $(n=142)$ were mild drinkers and $20.5 \%(n=229)$ were moderate drinkers. Table 1 summarizes the baseline clinical characteristics of the cohort. Among the patients in the smoker and non-smoker groups, fewer women were smokers; a history of atrial fibrillation was more common among the heavy smokers; higher blood pressure and circulating creatinine levels and a greater $P$ duration and left ventricle diameter were observed in the mild-tomoderate smokers and heavy smokers; and a larger right ventricle and left atrium diameter were observed in the heavy smokers. Among the patients in the non-drinker, mild-drinker and moderate-drinker categories, the number of women who were drinkers was lower, and a more frequent history of arterial hypertension, higher blood pressure levels and a larger left ventricle and left atrium diameter were observed in the moderate drinkers. There was no significant difference in terms of drug treatment at admission among the smokers or drinkers.

\section{Relationship between age, gender and all-cause mortality}

Of the 1118 patients studied, $262(23.4 \%)$ died and 3 $(0.26 \%)$ underwent heart transplantation during the mean follow-up of $3.5 \pm 2.3$ years. The all-cause mortality rates showed no difference between the non-smoker, mild-to-moderate-smoker and heavy-smoker groups (23.8, 20.8 and $24 \%$, respectively; log-rank $\chi^{2}=1.281$, $P=0.527)$. There was also no significant difference in mortality between the non-drinker, mild-drinker and moderate-drinker groups $(23.7,23.2$ and $22.3 \%$, respectively; log-rank $\chi^{2}=2.343, P=0.310$ ) (Fig. 2).

To determine whether smoking confers different degrees of risk between the different levels of drinkers with DCM, the entire cohort was divided into three subgroups and then further stratified according to the patients' status as non-smokers, mild-to-moderate smokers and heavy smokers. In the non-drinking patients, the all-cause mortality rates for non-smokers, mild-tomoderate smokers and heavy smokers were 24.3, 22.1 and $22.5 \%$, respectively; among the mild drinkers, the all-cause mortality rates for non-smokers, mild-to-moderate smokers and heavy smokers were 18.2, 17.9 and $28.6 \%$, respectively; and in the moderate drinkers, the all-cause mortality rates for non-smokers, mild-to-moderate smokers and heavy smokers were 18.9, 20.6 and $23.4 \%$, respectively. There was no significant difference in all-cause mortality between the subgroups of patients with different drinking statuses according their different smoking statuses (log-rank $\chi^{2}=1.286, P=0.526$ ) (Fig. 3).

\section{Cox proportional hazard models}

When the clinical, laboratory, electrograph and electrocardiographic data were considered, univariate analysis revealed that age, history of hypertension, ventricular premature beat, New York Heart Association (NYHA) functional class, systolic blood pressure, diastolic blood pressure, $P$ duration, QRS duration, left ventricle diameter, LVEF, right ventricle diameter, left atrium diameter, NT-pro-BNP, serum bilirubin, blood urea nitrogen, creatinine and fasting blood glucose were significant predictors of all-cause mortality in patients with DCM. Neither the smoking (HR 0.971, $P=0.663$ ) nor the drinking status (HR 0.891, $P=0.140$ ) was included in the Cox analysis. After adjustment for age, gender, smoking and drinking status, disease course, right ventricle and left atrium diameter, LVEF and serum creatinine, Cox multivariate analysis showed that ventricular premature beats, systolic blood pressure at admission, QRS duration, left atrium diameter and fasting blood glucose were powerful independent predictors of all-cause mortality in patients with DCM. Neither smoking nor drinking was found to be a predictor of all-cause mortality in the present study (Table 2).

\section{Discussion}

In this large-scale sample cohort study, we investigated the associations between smoking, drinking and all-cause mortality in patients with DCM. Our findings suggested that there was no predictive value of smoking and drinking for all-cause mortality in DCM patients; neither smoking nor drinking was an independent predictor of the all-cause mortality in patients with DCM.

Previous studies have found that that heavy drinkers exhibit a lower ejection fraction, greater enddiastolic volume, increased left atrial dimensions and increased left ventricular wall thickness, which occur in a 
Table 1 Patient characteristics categorized by ventricular conduction blockage patterns

\begin{tabular}{|c|c|c|c|c|c|c|c|c|c|}
\hline & $\begin{array}{l}\text { All patients } \\
(n=1118)\end{array}$ & $\begin{array}{l}\text { Non-smokers } \\
(n=593)\end{array}$ & $\begin{array}{l}\text { Moderate } \\
\text { smokers } \\
(n=159)\end{array}$ & $\begin{array}{l}\text { Heavy } \\
\text { smokers } \\
(n=366)\end{array}$ & $P$ value & $\begin{array}{l}\text { Non-drinkers } \\
(n=747)\end{array}$ & $\begin{array}{l}\text { Mild drinkers } \\
(n=142)\end{array}$ & $\begin{array}{l}\text { Moderate } \\
\text { drinkers } \\
(n=229)\end{array}$ & $P$ value \\
\hline Age (years) & $51.2 \pm 14.6$ & $51.0 \pm 16.4$ & $49.6 \pm 13.9$ & $52.1 \pm 11.5$ & 0.184 & $51.4 \pm 15.8$ & $50.4 \pm 13.0$ & $50.7 \pm 11.2$ & 0.650 \\
\hline $\begin{array}{l}\text { Female gender, } \\
n(\%)\end{array}$ & $300(26.8)$ & $280(47.2)$ & $13(8.2)$ & $7(1.9)$ & $<0.001$ & $293(39.2)$ & $4(2.8)$ & $3(1.3)$ & $<0.001$ \\
\hline \multicolumn{10}{|l|}{ History } \\
\hline $\begin{array}{l}\text { Disease dura- } \\
\text { tion (years) }\end{array}$ & $2(0.5-6)$ & $3(0.65-7)$ & $2(0.25-6)$ & $3(0.5-6)$ & 0.167 & $2(0.5-6)$ & $2(0.325-5)$ & $3(0.93-7)$ & 0.224 \\
\hline $\begin{array}{l}\text { Arterial } \\
\text { hyperten- } \\
\text { sion, } n \\
(\%)\end{array}$ & $300(26.8)$ & $142(23.9)$ & $48(30.2)$ & $110(30.1)$ & 0.068 & $178(23.8)$ & $41(28.9)$ & $81(35.4)$ & 0.002 \\
\hline $\begin{array}{l}\text { Diabetes } \\
\text { mellitus, } \\
n(\%)\end{array}$ & $161(14.4)$ & $84(14.2)$ & $23(14.5)$ & $54(14.8)$ & 0.968 & $108(14.5)$ & $18(12.7)$ & $35(15.3)$ & 0.783 \\
\hline Stroke, $n(\%)$ & $51(4.6)$ & $27(4.6)$ & $6(3.8)$ & $18(4.9)$ & 0.846 & $38(5.1)$ & $5(3.5)$ & $8(3.5)$ & 0.490 \\
\hline $\begin{array}{l}\text { Atrial fibrilla- } \\
\text { tion, } n(\%)\end{array}$ & $260(23.3)$ & $125(21.1)$ & $32(20.1)$ & $103(28.1)$ & 0.025 & $165(22.1)$ & $32(22.5)$ & $63(27.5)$ & 0.231 \\
\hline $\begin{array}{l}\text { Ventricular } \\
\text { premature } \\
\text { beat, } n(\%)\end{array}$ & $377(33.7)$ & 199 (33.6) & $54(34.0)$ & $124(33.9)$ & 0.992 & $253(33.9)$ & $51(35.9)$ & $73(31.9)$ & 0.718 \\
\hline $\begin{array}{l}\text { Ventricular } \\
\text { tachycardia, } \\
n(\%)\end{array}$ & 216 (19.3) & $120(20.2)$ & $24(15.1)$ & $72(19.7)$ & 0.338 & $152(20.3)$ & $26(18.3)$ & $38(16.6)$ & 0.429 \\
\hline $\begin{array}{l}\text { NYHA class } \\
\text { III and IV, } \\
n(\%)\end{array}$ & $821(73.4)$ & $439(74.0)$ & $106(66.7)$ & $276(75.4)$ & 0.102 & $558(74.7)$ & $99(69.7)$ & $164(71.6)$ & 0.367 \\
\hline \multicolumn{10}{|c|}{ Admission vital signs } \\
\hline $\mathrm{SBP}(\mathrm{mm} \mathrm{Hg})$ & $113.1 \pm 17.8$ & $111.3 \pm 17.4$ & $115.0 \pm 16.2$ & $115.4 \pm 18.7$ & 0.001 & $112.0 \pm 17.7$ & $115.8 \pm 17.4$ & $115.1 \pm 17.8$ & 0.012 \\
\hline $\mathrm{DBP}(\mathrm{mm} \mathrm{Hg})$ & $72.5 \pm 12.6$ & $71.0 \pm 12.3$ & $75.2 \pm 12.2$ & $73.6 \pm 13.0$ & $<0.001$ & $71.3 \pm 12.6$ & $74.2 \pm 12.9$ & $75.1 \pm 12.2$ & $<0.001$ \\
\hline $\begin{array}{l}\text { Heart rate, } \\
\text { beats/min }\end{array}$ & $80.8 \pm 17.4$ & $81.3 \pm 17.8$ & $79.9 \pm 16.8$ & $80.8 \pm 17.4$ & 0.611 & $81.1 \pm 17.4$ & $79.3 \pm 17.4$ & $80.9 \pm 16.5$ & 0.546 \\
\hline \multicolumn{10}{|c|}{ Laboratory values at admission } \\
\hline AST (IU/L) & $26(20-35)$ & $25(19-34.75)$ & $28(20.75-36)$ & $25(20-35)$ & 0.969 & $26(19-35)$ & $25(20-34)$ & $27(21-37)$ & 0.422 \\
\hline ALT (IU/L) & $28(19-45)$ & $26(18-43)$ & $33(22-58.5)$ & $30(20-45.5)$ & 0.826 & $27(19-44)$ & $28(20-45)$ & $32(22-47.75)$ & 0.656 \\
\hline TB $(\mathrm{mmol} / \mathrm{L})$ & $\begin{array}{c}20.3(15.1- \\
30.625)\end{array}$ & $\begin{array}{l}20.5(15.1- \\
31.5)\end{array}$ & $\begin{array}{c}19(15.175- \\
28.525)\end{array}$ & $\begin{array}{l}20.3(15.1- \\
29.5)\end{array}$ & 0.738 & $\begin{array}{l}20.2 \\
\quad(15-30.775)\end{array}$ & $\begin{array}{l}19.9(16.2- \\
29.35)\end{array}$ & $21(14.85-30.3)$ & 0.834 \\
\hline $\mathrm{DB}(\mathrm{mmol} / \mathrm{L})$ & $3.7(2.5-6.575)$ & $3.6(2.4-6.9)$ & $3.5(2.5-5.775)$ & $3.8(2.6-6.25)$ & 0.653 & $3.7(2.4-6.8)$ & $3.6(2.5-6.2)$ & $\begin{array}{l}3.85 \\
(2.5-6.575)\end{array}$ & 0.869 \\
\hline $\begin{array}{l}\text { Glucose } \\
\text { (mmol/L) }\end{array}$ & $5.61 \pm 1.83$ & $5.61 \pm 1.90$ & $5.71 \pm 2.04$ & $5.58 \pm 1.58$ & 0.763 & $5.61 \pm 1.83$ & $5.48 \pm 2.04$ & $5.67 \pm 1.56$ & 0.614 \\
\hline $\mathrm{TG}(\mathrm{mmol} / \mathrm{L})$ & $1.56 \pm 1.02$ & $1.57 \pm 1.06$ & $1.60 \pm 0.94$ & $1.53 \pm 0.98$ & 0.736 & $1.54 \pm 1.04$ & $1.50 \pm 0.81$ & $1.67 \pm 1.05$ & 0.237 \\
\hline $\begin{array}{l}\mathrm{CHO} \\
(\mathrm{mmol} / \mathrm{L})\end{array}$ & $4.60 \pm 1.11$ & $4.56 \pm 1.12$ & $4.61 \pm 1.14$ & $4.66 \pm 1.10$ & 0.458 & $4.58 \pm 1.12$ & $4.62 \pm 1.05$ & $4.67 \pm 1.20$ & 0.545 \\
\hline $\begin{array}{c}\text { Creatinine } \\
(\mu \mathrm{mol} / \mathrm{L})\end{array}$ & $92.8 \pm 35.3$ & $88.2 \pm 34.7$ & $98.2 \pm 44.4$ & $97.8 \pm 30.6$ & $<0.001$ & $91.2 \pm 38.2$ & $94.7 \pm 25.7$ & $96.9 \pm 29.9$ & 0.082 \\
\hline BUN $(\mu \mathrm{mol} / \mathrm{L})$ & $7.98 \pm 3.99$ & $7.79 \pm 3.71$ & $8.15 \pm 5.62$ & $8.20 \pm 3.56$ & 0.264 & $7.96 \pm 4.06$ & $7.63 \pm 2.63$ & $8.24 \pm 4.45$ & 0.366 \\
\hline $\begin{array}{l}\text { Pro-NT BNP } \\
(\mathrm{fmol} / \mathrm{ml})\end{array}$ & $\begin{array}{l}1534.85 \\
(790.725- \\
2795.15)\end{array}$ & $\begin{array}{l}1543.4 \\
(828.85- \\
2827.05)\end{array}$ & $\begin{array}{l}1475.55 \\
(701.05- \\
2661.425)\end{array}$ & $\begin{array}{l}1547.2 \\
(790.35- \\
2826.55)\end{array}$ & 0.353 & $\begin{array}{l}1583(825.3- \\
2907.2)\end{array}$ & $\begin{array}{l}1420(721.8- \\
2898.3)\end{array}$ & $\begin{array}{c}1399.6(737.3- \\
2559.75)\end{array}$ & 0.149 \\
\hline \multicolumn{10}{|c|}{ Electrograph data } \\
\hline $\begin{array}{l}\text { QRS duration } \\
(\mathrm{ms})\end{array}$ & $119.7 \pm 30.9$ & $119.9 \pm 32.4$ & $119.7 \pm 27.9$ & $119.2 \pm 29.9$ & 0.941 & $119.6 \pm 30.9$ & $119.1 \pm 31.0$ & $120.0 \pm 30.8$ & 0.963 \\
\hline QT (ms) & $405.8 \pm 54.5$ & $406.7 \pm 55.7$ & $406.9 \pm 57.5$ & $403.9 \pm 51.1$ & 0.727 & $406.4 \pm 54.1$ & $403.0 \pm 47.9$ & $404.8 \pm 58.7$ & 0.768 \\
\hline$P(\mathrm{~ms})$ & $107.5 \pm 21.7$ & $105.0 \pm 21.1$ & $110.0 \pm 20.6$ & $110.7 \pm 22.8$ & 0.002 & $106.3 \pm 21.8$ & $108.2 \pm 20.3$ & $111.4 \pm 21.8$ & 0.036 \\
\hline $\mathrm{PR}(\mathrm{ms})$ & $183.0 \pm 33.0$ & $183.0 \pm 33.4$ & $178.2 \pm 32.6$ & $185.2 \pm 32.4$ & 0.148 & $183.0 \pm 33.8$ & $182.5 \pm 32.8$ & $183.5 \pm 30.6$ & 0.965 \\
\hline
\end{tabular}


Table 1 continued

\begin{tabular}{|c|c|c|c|c|c|c|c|c|c|}
\hline & $\begin{array}{l}\text { All patients } \\
(n=1118)\end{array}$ & $\begin{array}{l}\text { Non-smokers } \\
(n=593)\end{array}$ & $\begin{array}{l}\text { Moderate } \\
\text { smokers } \\
(n=159)\end{array}$ & $\begin{array}{l}\text { Heavy } \\
\text { smokers } \\
(n=366)\end{array}$ & $P$ value & $\begin{array}{l}\text { Non-drinkers } \\
(n=747)\end{array}$ & $\begin{array}{l}\text { Mild drinkers } \\
(n=142)\end{array}$ & $\begin{array}{l}\text { Moderate } \\
\text { drinkers } \\
(n=229)\end{array}$ & $P$ value \\
\hline \multicolumn{10}{|c|}{ Echocardiography data } \\
\hline $\mathrm{LVd}(\mathrm{mm})$ & $68.1 \pm 9.4$ & $67.3 \pm 9.15$ & $69.2 \pm 9.10$ & $69.1 \pm 9.76$ & 0.004 & $67.6 \pm 9.27$ & $69.6 \pm 9.80$ & $69.0 \pm 9.40$ & 0.020 \\
\hline $\operatorname{LVEF}(\mathrm{mm})$ & $31.9 \pm 8.4$ & $31.5 \pm 8.0$ & $32.1 \pm 8.9$ & $32.3 \pm 8.7$ & 0.341 & $31.6 \pm 8.2$ & $32.1 \pm 8.6$ & $32.7 \pm 9.0$ & 0.232 \\
\hline $\mathrm{RV}(\mathrm{mm})$ & $23.6 \pm 5.4$ & $23.3 \pm 5.2$ & $23.0 \pm 4.7$ & $24.5 \pm 5.8$ & 0.004 & $23.5 \pm 5.4$ & $23.9 \pm 4.9$ & $23.8 \pm 5.7$ & 0.583 \\
\hline $\mathrm{LA}(\mathrm{mm})$ & $44.0 \pm 7.7$ & $43.2 \pm 7.6$ & $43.9 \pm 7.9$ & $45.4 \pm 7.6$ & $<0.001$ & $43.6 \pm 7.5$ & $44.7 \pm 8.6$ & $45.1 \pm 7.5$ & 0.020 \\
\hline \multicolumn{10}{|c|}{ Medications at admission } \\
\hline $\begin{array}{l}\text { Diuretics, } n \\
(\%)\end{array}$ & 1085 (94.6) & $562(94.8)$ & $151(95.0)$ & $345(94.3)$ & 0.925 & $706(94.5)$ & $130(91.5)$ & $222(96.9)$ & 0.079 \\
\hline $\begin{array}{l}\text { ACEI/ARB, } n \\
\text { (\%) }\end{array}$ & $947(84.7)$ & $500(84.3)$ & $137(86.2)$ & $310(84.7)$ & 0.848 & $623(83.4)$ & $125(88.0)$ & 199 (86.9) & 0.218 \\
\hline $\begin{array}{l}\text { Beta-blockers, } \\
n(\%)\end{array}$ & 1015 (90.8) & $537(90.6)$ & $151(95.0)$ & $327(89.3)$ & 0.118 & 677 (90.6) & $129(90.8)$ & 209 (91.3) & 0.958 \\
\hline Digoxin, $n(\%)$ & $898(80.3)$ & $486(82.0)$ & $124(78.0)$ & $288(78.7)$ & 0.593 & $587(78.6)$ & $118(83.1)$ & $193(84.3)$ & 0.111 \\
\hline $\begin{array}{l}\text { Spironolac- } \\
\text { tone, } n(\%)\end{array}$ & $1016(90.9)$ & $544(91.7)$ & $142(89.3)$ & $330(90.2)$ & 0.542 & $680(91.0)$ & $125(88.0)$ & $211(92.1)$ & 0.396 \\
\hline
\end{tabular}

Data are expressed as mean \pm SD, medians (interquartile range) or percentages; $P$ values from independent-sample $t$ tests are shown Italics indicate $P<0.05$

1 patient lacked drinking status data; 22 lacked electrocardiogram data; 43 lacked echocardiography data; 334 lacked NT-pro-BNP levels; 47 lacked fasting blood glucose levels; 29 lacked creatinine data; 40 lacked BUN levels; 83 lacked triglyceride data and total cholesterol levels; 71 lacked AST data; 72 lacked ALT data; 72 lacked TB data; 74 lacked DB data; and 17 lacked data on the medications at admission

NYHA New York Heart Association, SBP systolic blood pressure, DBP diastolic blood pressure, AST aspartate aminotransferase, $A L T$ alanine aminotransferase, BUN blood urea nitrogen, $T G$ triglyceride, $T C$ total cholesterol, $B U N$ blood urea nitrogen, $T B$ total bilirubin, $D B$ direct bilirubin, $B U N$ blood urea nitrogen, $N T$-pro- $B N P N$-terminal fragment pro-brain natriuretic peptide, $L V$ left ventricle, $L A$ left atrium, $L V E F$ left ventricular ejection fraction, $A C E I$ angiotensin-converting enzyme inhibitor, $A R B$ angiotensin receptor blocker

Table 2 Cox regression of all-cause mortality in patients with DCM

\begin{tabular}{|c|c|c|c|c|c|c|}
\hline \multirow[t]{2}{*}{ Variable } & \multicolumn{3}{|c|}{ Univariate analysis } & \multicolumn{3}{|c|}{ Multivariate analysis } \\
\hline & HR & $95 \% \mathrm{Cl}$ & $P$ value I & HR & $95 \% \mathrm{Cl}$ & $P$ value \\
\hline Age & 1.010 & $1.001-1.019$ & 0.024 & 1.004 & $0.991-1.016$ & 0.558 \\
\hline Sex & 1.121 & $0.855-1.469$ & 0.408 & 1.275 & $0.871-1.867$ & 0.212 \\
\hline Ventricular premature beat & 1.462 & $1.145-1.868$ & 0.002 & 1.358 & $1.020-1.807$ & 0.036 \\
\hline NYHA functional class & 1.592 & $1.350-1.877$ & $<0.001$ & 1.223 & $0.990-1.511$ & 0.062 \\
\hline Disease duration & 1.028 & $1.011-1.044$ & 0.001 & 1.012 & $0.990-1.035$ & 0.291 \\
\hline Smoker & 0.908 & $0.712-1.158$ & 0.436 & 0.911 & $0.649-1.279$ & 0.591 \\
\hline Drinker & 0.817 & $0.630-1.060$ & 0.129 & 0.951 & $0.669-1.352$ & 0.779 \\
\hline Systolic blood pressure & 0.982 & $0.975-0.989$ & $<0.001$ & 0.984 & $0.975-0.994$ & 0.001 \\
\hline QRS duration & 1.009 & $1.006-1.013$ & $<0.001$ & 1.010 & $1.005-1.014$ & $<0.001$ \\
\hline Left ventricle & 1.037 & $1.025-1.050$ & $<0.001$ & 1.003 & $0.983-1.022$ & 0.796 \\
\hline Right ventricle & 1.061 & $1.037-1.086$ & $<0.001$ & 1.015 & $0.986-1.045$ & 0.328 \\
\hline Left atrium & 1.055 & $1.040-1.071$ & $<0.001$ & 1.047 & $1.025-1.069$ & $<0.001$ \\
\hline LVEF & 0.963 & $0.948-0.978$ & $<0.001$ & 0.980 & $0.961-1.001$ & 0.059 \\
\hline NT-pro-BNP & 1.045 & $1.024-1.067$ & $<0.001$ & & & \\
\hline FBG & 1.097 & $1.041-1.156$ & $<0.001$ & 1.097 & $1.026-1.173$ & 0.006 \\
\hline Creatinine & 1.004 & $1.002-1.007$ & 0.001 & 1.003 & $1.000-1.006$ & 0.053 \\
\hline
\end{tabular}

The variables analyzed in the multivariate Cox mode included age, gender, ventricular premature beat, drinking and smoke status, disease duration, NYHA functional classes, systolic blood pressure, QRS duration, left ventricular, right ventriclular and left atrium diameter, LVEF, FBG and creatinine

Italics indicate $P<0.05$ 


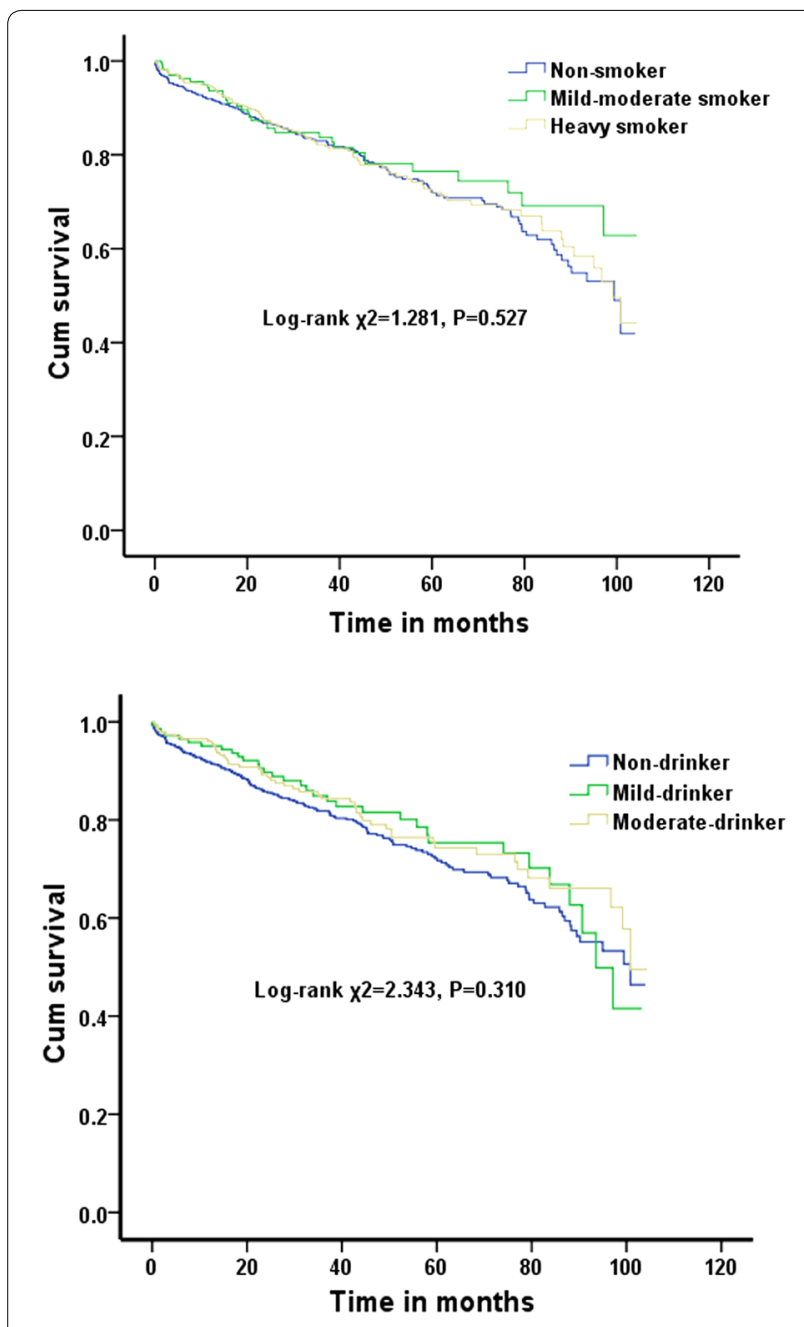

Fig. 2 Kaplan-Meier survival curves for the smoking- and drinkingrelated groups of patients with dilated cardiomyopathy. The upper panel shows the survival curves among the non-smoker, mild-tomoderate-smoker and heavy-smoker groups (23.8, 20.8 and $24 \%$; log-rank $\left.\chi^{2}=1.281, P=0.527\right)$. The lower panel shows the survival curves among the non-drinker, mild-drinker and moderate-drinker groups $\left(23.7,23.2\right.$ and $22.3 \%$; log-rank $\left.\chi^{2}=2.343, P=0.310\right)$

dose-dependent fashion and precede the onset of clinical symptoms or physical findings [28, 29]. The mechanisms underlying alcohol-induced myocardial damage include cardiac myocyte apoptosis [30], alterations in the excitation-contraction coupling in cardiac myocytes [31], and increased oxidative stress [32] and activation of the renin-angiotensin system and the sympathetic nervous system [33]. However, moderate alcohol consumption has been proposed to confer protection against cardiovascular events while also increasing high-density lipoprotein cholesterol, decreasing platelet aggregation and coagulation, enhancing endothelial function, reducing inflammation, promoting antioxidant effects and decreasing the

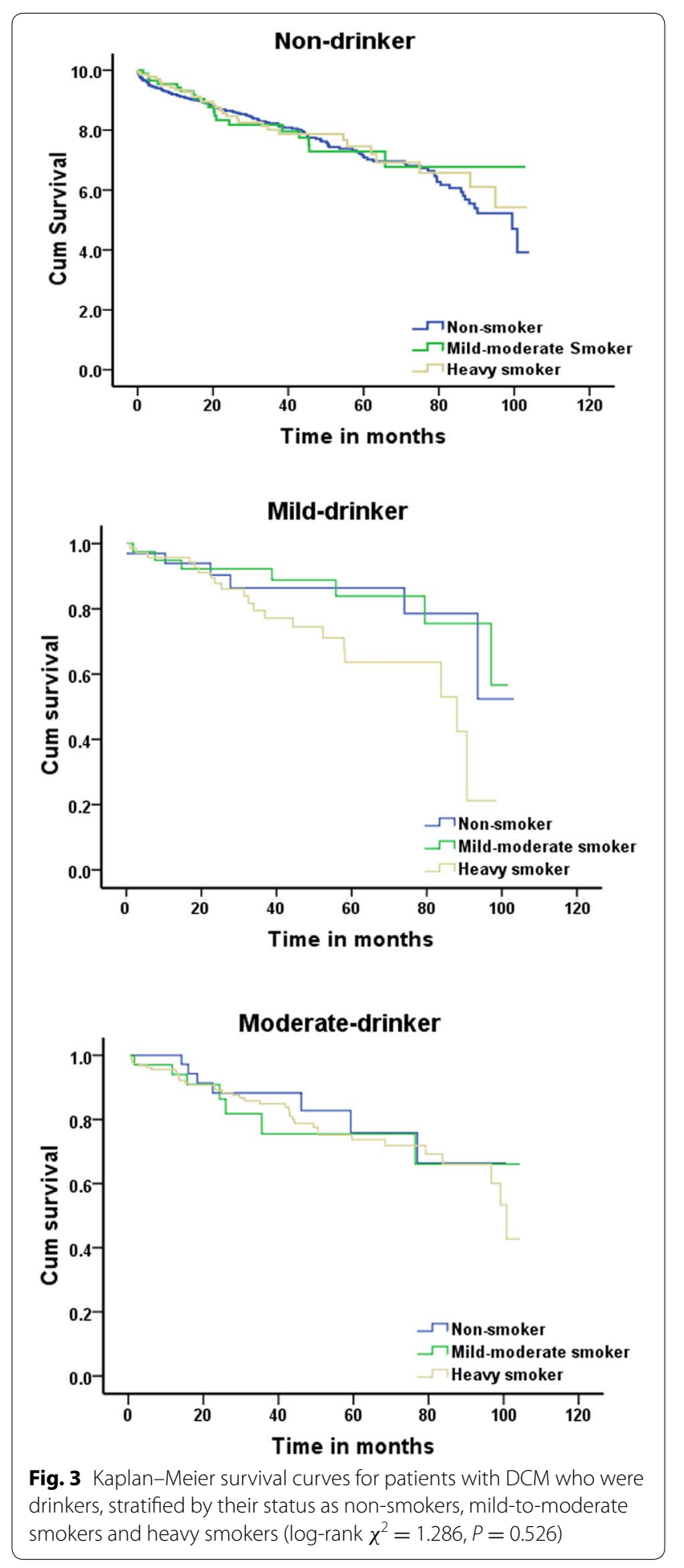

activity of angiotensin II (Ang II) [34-36]. In the present large-sample cohort study, although heavy drinkers were excluded owing to alcoholic cardiomyopathy, no significant differences were found between patients who were 
non-drinkers, mild drinkers and moderate drinkers. In a study in patients with a previous myocardial infarction, those who consumed small-to-moderate amounts of alcohol exhibited a lower total mortality [10]. However, the present study found no favorable effect of mild-tomoderate drinking on the all-cause mortality in the DCM patients.

Tobacco smoking has been solidly implicated in the etiology of cardiovascular diseases such as coronary artery disease, aortic aneurysm, stroke and peripheral vascular diseases [37], as cigarette smoking has been associated with higher serum levels of cholesterol, coronary vasomotor reactivity, platelet aggregation, and a prothrombotic state [38-41]. Additionally, an association between smoking and cardiomyopathy has been suggested by the results of several animal studies [42-44]. Possible mechanisms underlying the association between smoking and cardiomyopathy include direct damage to cardiac muscles (following damage to the myocardial mitochondria) and an increase in cardiac susceptibility to viral infections [43, 45]. Although it is well known that smoking increases cardiovascular morbidity and mortality $[15,16]$, the effect of smoking on the mortality of DCM patients cannot be conclusively determined based on the available data [19-22]. In the present study, there was no detectable influence of mild-to-moderate smoking and heavy smoking on the all-cause mortality in DCM patients. Although the allcause mortality rate was lower among mild-to-moderate smokers than among non-smokers, this association did not achieve statistical significance.

\section{Conclusion}

In conclusion, there were no significant differences in mortality between the smoking- and drinking-related patient groups, indicating no effect of smoking and drinking on all-cause mortality in patients with DCM in the present large-scale study.

\section{Limitations}

The present study has several limitations. Like all hospital-based cohorts, the examined study population was a selected population of patients who had been referred for treatment. Because the NT-pro-BNP test was not commonly used until the later years of this study and its results were missing in 334 patients, we excluded NTpro-BNP data from the multivariate Cox analysis to avoid the inclusion of potential confounding variables in the statistical analyses. Additionally, $14.1 \%$ of the patients ( $n=158$ ) were lost to follow-up because of factors such as a lack of communication in rural areas; however, the main outcomes were not changed when we detected the losers in the Kaplan-Meier survival and Cox analyses. Ideally, all patients with DCM should be confirmed to be free of coronary artery disease. In practice, however, coronary arteriography is not routinely performed in all patients with congestive heart failure. Because retrospective studies cannot control the conditions under which patients are recruited or investigated, there was a very small subgroup of ischemic heart disease patients in the present study compared with the expected rates based on the numbers of patients who were subjected to coronary artery angiography, coronary CT scans or cardiac radionuclide imaging at other hospitals, as only 334 patients underwent coronary artery angiography and only 80 showed positive results at our hospital.

\section{Abbreviations}

DCM: dilated cardiomyopathy; NYHA: New York Heart Association; SBP: systolic blood pressure; DBP: diastolic blood pressure; AST: aspartate aminotransferase; ALT: alanine aminotransferase; BUN: blood urea nitrogen; TG: triglyceride; TC: total cholesterol; BUN: blood urea nitrogen; TB: total bilirubin; DB: direct bilirubin; BUN: blood urea nitrogen; NT-pro-BNP: N-terminal fragment pro-brain natriuretic peptide; LV: left ventricle; LA: left atrium; LVEF: left ventricular ejection fraction; $A C E l$ : angiotensin-converting enzyme inhibitor; ARB: angiotensin receptor blocker; Cl: confidence interval.

\section{Authors' contributions}

$\mathrm{XL}, \mathrm{GL}$ and $\mathrm{ML}$ conducted the patients' enrollment, data collection and followup work. YL, RL and PL participated in the data collection and performed the statistical analysis. WH and TH conceived the study and participated in its design and coordination; they also helped to draft the manuscript. All authors read and approved the final manuscript.

\section{Author details \\ ${ }^{1}$ Department of Cardiology, Sichuan Academy of Medical Sciences and Sichuan Provincial People's Hospital, Chengdu 610072, Sichuan, People's Republic of China. ${ }^{2}$ School of Medicine, University of Electronic Science and Technology of China, Chengdu 610072, Sichuan, People's Republic of China. ${ }^{3}$ State Key Laboratory of Cardiovascular Disease, Cardiac Arrhyth- mia Center, Fuwai Hospital, National Center for Cardiovascular Diseases, Chinese Academy of Medical Sciences and Peking Union Medical College, Beijing 100037, People's Republic of China. ${ }^{4}$ Department of Cardiac Surgery, Beijing Anzhen Hospital, Capital Medical University, Beijing 100029, People's Republic of China. ${ }^{5}$ Key Laboratory of Thermoregulation and Inflamma- tion of Sichuan Higher Education Institutes, Chengdu Medical College, Chengdu 610500, People's Republic of China. ${ }^{6}$ Cardiovascular Institute and Fuwai Hospital, Chinese Academy of Medical Sciences, Peking Union Medical College, Beijing 100037, China. ${ }^{7}$ Hospital of the University of Elec- tronic Science and Technology of China and Sichuan Provincial People's Hospital, Chengdu 610072, China.}

\section{Acknowledgements}

This study was supported by grants from the National Natural Science Foundation of China (nos. 81000104, 81160141, and 81470521).

\section{Compliance with ethical guidelines}

\section{Competing interests}

The authors declare that they have no competing interests.

Received: 30 March 2015 Accepted: 4 September 2015

Published online: 17 September 2015 


\section{References}

1. Richardson P, McKenna W, Bristow M, Maisch B, Mautner B, O'Connell J, Olsen E, Thiene G, Goodwin J, Gyarfas I, Martin I, Nordet P. Report of the 1995 World Health Organization/International Society and Federation of Cardiology Task Force on the Definition and Classification of Cardiomyopathies. Circulation. 1996;93:841-2

2. Katz AM. Cardiomyopathy of overload. A major determinant of prognosis in congestive heart failure. N Engl J Med. 1990;322:100-10.

3. Kupari M, Koskinen P, Suokas A. Left ventricular size, mass and function in relation to the duration and quantity of heavy drinking in alcoholics. Am J Cardiol. 1991;67:274-9.

4. McKenna CJ, Codd MB, McCann HA, Sugrue DD. Alcohol consumption idiopathic dilated cardiomyopathy: a case control study. Am Heart J. 1998;135:833-7

5. Costanzo S, Di Castelnuovo A, Donati MB, lacoviello L, de Gaetano G. Recent advances on the relationship between alcohol consumption and cardiovascular mortality. Recenti Prog Med. 2011;102(3):105-8.

6. Rehm J, Sempos CT. Alcohol consumption and all-cause mortality. Addiction. 1995;90:471-80.

7. Fillmore K, Kerr WC, Bostrom A. Changes in drinking status, serious illness and mortality. J Stud Alcohol. 2003;64:278-85.

8. Bos S, Grobbee DE, Boer JMA, Verschuren WM, Beulens JWJ. Alcohol consumption and risk of cardiovascular disease among hypertensive women. Eur J Cardiovasc Prev Rehabil. 2010;17:119-26.

9. Abramson JL, Williams ZA, Krumholz HM, Vaccarino V. Moderate alcohol consumption and risk of heart failure among older persons. JAMA. 2001;285:1971-7.

10. Muntwyler J, Hennekens CH, Buring JE, Gaziano JM. Mortality and light to moderate alcohol consumption after myocardial infarction. Lancet. 1998;352(9144):1882-5

11. Beulens JWJ, Algra A, Soedamah-Muthu SS, Visseren FL, Grobbee DE van der Graaf Y. Alcohol consumption and risk of recurrent cardiovascular events and mortality in patients with clinically manifest vascular disease and diabetes mellitus: the Second Manifestations of Arterial (SMART) disease study. Atherosclerosis. 2010;212:281-6.

12. Thun MJ, Peto R, Lopez AD, Monaco JH, Henley SJ, Heath CW, Doll R. Alcohol consumption and mortality among middle-aged and elderly US adults. New Engl J Med. 1997;337:1705-14.

13. Ockene IS, Miller NH. Cigarette smoking, cardiovascular disease, and stroke: a statement for healthcare professionals from The American Heart Association. Circulation. 1997;96:3243-7.

14. He J, Ogden LG, Bazzano LA, Vupputuri S, Loria C, Whelton PK. Risk factors for congestive heart failure in US men and women: NHANES I epidemiologic follow-up study. Arch Intern Med. 2001;161:996-1002.

15. National Cancer Institute: Health Effects Of Exposure To Environmental Tobacco Smoke: The Report Of The California Environmental Protection Agency, Smoking And Tobacco Control Monograph No. 10. NIH Pub. No. 99-4645. Bethesda, MD: Department Of Health And Human Services, National Institutes Of Health, National Cancer Institute, 1999.

16. US Department Of Health And Human Services. The health consequences of smoking. A report of the surgeon general. Atlanta: Centers For Disease Control And Prevention, National Center For Chronic Disease Prevention And Health Promotion, Office On Smoking And Health, 2004

17. Hartz AJ, Anderson AJ, Brooks HL, Manley JC, Parent GT, Barboriak JJ. The association of smoking with cardiomyopathy. N Engl J Med. 1984;311(19):1201-6.

18. Hartz AJ, Ratner ER, Sinoway LI, Bartholomew MJ. Smoking and idiopathic congestive cardiomyopathy. Jpn Heart J. 1996:37(3):401-7.

19. Juillière $Y$, Danchin $N$, Briançon $S$, et al. Dilated cardiomyopathy: long-term follow- up and predictors of survival. Int J Cardiol. 1988;21(3):269-77.

20. Juillière Y, Danchin N, Briançon S, Khalife K, Balaud A, Gilgenkrantz JM, Pernot C, Cherrier F. Survival in primary dilated cardiomyopathy as a function of tobacco smoking. A retrospective study. Presse Med. 1989;18(16):809-12

21. Metayer C, Coughlin SS, Mather FJ. Does cigarette smoking paradoxically increase survival in idiopathic dilated cardiomyopathy? The Washington, D.C., Dilated Cardiomyopathy Study. Cardiology. 1996;87(6):502-8.
22. Ikram H, Williamson HG, Won M, Crozier IG, Wells EJ. The course of idiopathic dilated cardiomyopathy in New Zealand. Br Heart J. 1987;57(6):521-7

23. The WHO/ISFC Task Force. Report of the WHO/ISFC task force on the definition and classification of cardiomyopathies. Br Heart J 1980;44:672-3.

24. Rostron B. Alcohol consumption and mortality risks in the USA. Alcohol Alcohol. 2012:47(3):334-49.

25. Li Y, Swensen SJ, Karabekmez LG, Marks RS, Stoddard SM, Jiang R, Worra JB, Zhang F, Midthun DE, de Andrade M, Song Y, Yang P. Effect of emphysema on lung cancer risk in smokers: a computed tomography-based assessment. Cancer Prev Res. 2011:4(1):43-50.

26. Forey BA, Thornton AJ, Lee PN. Systematic review with meta-analysis of the epidemiological evidence relating smoking to COPD, chronic bronchitis and emphysema. BMC Pulm Med. 2011;11:36.

27. Lang RM, Bierig M, Devereux RB, Flachskampf FA, Foster E, Pellikka PA, Picard MH, Roman MJ, Seward J, Shanewise JS, Solomon SD, Spencer KT, Sutton MS, Stewart WJ. Recommendations for chamber quantification: a report from the American Society of Echocardiography's Guidelines and Standards Committee and the Chamber Quantification Writing Group, developed in conjunction with the European Association of Echocardiography, a branch of the European Society of Cardiology. J Am Soc Echocardiogr. 2005;18:1440-63.

28. Urbano-Marquez A, Estruch R, Navarro-Lopez F, Grau JM, Mont L, Rubin $E$. The effects of alcoholism on skeletal and cardiac muscle. New Engl J Med. 1989;320:409-15.

29. Mathews EC Jr, Gardin JM, Henry WL, del Negro AA, Fletcher RD, Snow JA, Epstein SE. Echocardiographic abnormalities in chronic alcoholics with and without overt congestive heart failure. Am J Cardiol. 1981;47:570-8.

30. Haunstetter A, Izumo S. Apoptosis: basic mechanisms and implications for cardiovascular disease. Circ Res. 1998;82:1111-29.

31. Danziger RS, Sakai M, Capogrossi MC, Spurgeon HA, Hansford RG, Lakatta EG. Ethanol acutely and reversibly suppresses excitation-contraction coupling in cardiac myocytes. Circ Res. 1991;68:1660-8.

32. Capasso JM, Li P, Guideri G, Malhotra A, Cortese R, Anversa P. Myocardial mechanical, biochemical, and structural alterations induced by chronic ethanol ingestion in rats. Circ Res. 1992;71:346-56.

33. Cheng CP, Cheng HJ, Cunningham C, Shihabi ZK, Sane DC, Wannenburg T, Little WC. Angiotensin II type 1 receptor blockade prevents alcoholic cardiomyopathy. Circulation. 2006;114:226-36.

34. Gronbaek M, Deis A, Sorensen TI, Becker U, Schnohr P, Jensen G. Mortality associated with moderate intakes of wine, beer, or spirits. Br Med J. 1995:310:1165-9.

35. Saremi A, Arora R. The cardiovascular implications of alcohol and red wine. Am J Ther. 2008;15:265-77.

36. Opie LH, Lecour S. The red wine hypothesis: from concepts to protective signaling molecules. Eur Heart J. 2007:28:1683-93.

37. Wald NJ, Hackshaw AK. Cigarette smoking: an epidemiological overview. Br Med Bull. 1996;52:3-11.

38. Muscat JE, Harris RE, Haley NJ, Wynder EL. Cigarette smoking and plasma cholesterol. Am Heart J. 1991;121:141-7.

39. Hung J, Lam JY, Lacoste L, Letchacovski G. Cigarette smoking acutely increases platelet thrombus formation in patients with coronary artery disease taking aspirin. Circulation. 1995:92:2432-6.

40. Campisi R, Czernin J, Schöder H, Sayre JW, Marengo FD, Phelps ME, Schelbert HR. Effects of long-term smoking on myocardial blood flow, coronary vasomotion and vasodilator capacity. Circulation. 1998;98:119-25.

41. Fusegawa Y, Goto S, Handa S, Kawada T, Ando Y. Platelet spontaneous aggregation in platelet-rich plasma is increased in habitual smokers. Thromb Res. 1999;93:271-8.

42. Ahmed SS, Moschos CB, Oldewurtel HA, Regan TJ. Myocardial effects of long term cigarette smoking: relative roles of carbon monoxide and nicotine. Am J Cardiol. 1980:46:593-8.

43. Gvozdjáková A, Bada V, Sány L, Kucharská J, Krutý F, Bozek P, Trstanský L, Gvozdják J. Smoke cardiomyopathy: disturbance of oxidative processes in myocardial mitochondrial. Cardiovasc Res. 1984;18:229-32.

44. Lough J. Cardiomyopathy produced by cigarette smoke: ultrastructural observations in guinea pigs. Arch Pathol Lab Med. 1978;102:377-80.

45. Hayes JM, Hall GV. The myocardial toxicity of carbon monoxide. Med J Aust. 1964:1:865-8. 